\title{
Psychological Models of Depression and Anxiety: Counselor's Perspectives
}

\author{
Vijaya Lakshmi Chouhan ${ }^{1}$, Preeti Sharma ${ }^{2} *$
}

\section{ABSTRACT}

Negative psychological states like anxiety and depression have been the major focus of psychology over the last hundreds of years. People experience these negative psychological states as a part of their response to their threatening life events. These states sometimes help to cope with threatening situations. Humans are hard wired to response in these ways as they are adaptive subsequent to traumatic events. There are multiple psychological models which explain the occurrence of anxiety and depression in clinical depression. The understandings of the formulation of these models help in the management of these clinical conditions. Anxiety and depression often co-exist among patients. The understanding of co-morbidity of anxiety and depression is the key in proper diagnosis and management of these clinical states. The current paper will focus on the detail description of psychological models of anxiety and depression and the possible link between these two clinical conditions. The paper will also enumerate brief psychological strategies of how to deal with anxiety and depression.

Keywords: Anxiety, Depression, Psychological Models, Threat, Coping

Anxiety is defined as apprehension about some unknown phenomena. Constant worry is the basic feature of anxiety. The root of anxiety lies in unconscious conflict between id and superego. Anxiety involves state of apprehension. Unlike fear, where the cause is known, in case of anxiety cause is unknown. May (1950) defined anxiety as "the apprehension cued off by a threat to some value which the individual holds essential to his existence as personality" Anxiety symptoms can be broadly classified in four groups. Cognitive symptoms: Indecisiveness, worry, impaired attention and concentration, memory impairment; Affective/emotional symptoms: Nervousness, anger, sadness; Physical symptoms: Muscle tightness, disturbed sleep, body-aches, high blood pressure, dry mouth, etc; Behavioral Symptoms; Irritability, impulsivity etc.

\footnotetext{
${ }^{1}$ President, Academy of Well-being \& Former Dean, Students’ Welfare Board, MLSU, HOD, Department of Psychology, Udaipur, Rajasthan, India

${ }^{2}$ Counselor, Academy of Well-being, Udaipur \& Research Scholar, Pacific University, Udaipur, India

*Responding Author
}

Received: December 25, 2016; Revision Received: January 15, 2017; Accepted: January 27, 2017

(C) 2017 Chouhan V, Sharma P; licensee IJIP. This is an Open Access Research distributed under the terms of the Creative Commons Attribution License (creativecommons.org/licenses/by/2.0), which permits unrestricted use, distribution, and reproduction in any Medium, provided the original work is properly cited. 


\section{Psychological Models of Depression and Anxiety: Counselor's Perspectives}

\section{Neuroticism}

Neuroticism is a fundamental personality trait in the study of psychology. This trait is considered as proneness to anxiety. It can be defined as an enduring tendency to experience negative emotional states. It is featured in the form of constant worry, preoccupied that some bad will happen in future, and allows emotions to influence judgments. It was initially studied by Freud and later Eysenck included it in his personality model. The term neurosis was, however coined by Cullen in 1769 . He used the term to refer to "disorders of sense and motion" caused by a "general affection of the nervous system." For him, it described various nervous disorders and symptoms that could not be explained physiologically.

Individuals who score high on neuroticism are more likely than the average to experience such feelings as anxiety, anger, guilt, and clinical depression. They respond more poorly to environmental stress, and are more likely to interpret ordinary situations as threatening, and minor frustrations as hopelessly difficult. They are often self-conscious and shy, and they may have trouble controlling urges and delaying gratification. Neuroticism is related to emotional intelligence, which involves emotional regulation, motivation, and interpersonal skills. It is also considered to be a predisposition for traditional neuroses, such as phobias and other anxiety disorders.

\section{MODELS OF ANXIETY}

1. Psychodynamic model: According to psychodynamic model, Freud (1926) argues that anxiety emanates from an unpleasant affective state originating in the unconscious. The anxiety is a symbol or signal of an unconscious conflict, usually stemming from childhood that remains unresolved. To deal with their feelings of anxiety, people develop psychological defenses that may be either adaptive or maladaptive.

2. Attachment model: This model was given by Bowlby in 1969. The basic premise of this model is that insecure attachment between the care giver and the child is the main cause of anxiety disorders in the child.

3. Eysenck's arousal model: Eysenck (1968) posits that activity of the cortico-reticular loop is associated with increased cortical arousal. This system provides neural substrate for extroversion-introversion. It is hypothesized that this system is easily activated in the introverts than extroverts, so that introverts are more easily aroused and shows high level of cortical arousal.

4. Incubation model: Incubation model of anxiety was given by Eysenck in the 1968.The basic idea of this model is the phenomena of extinction that is a part of classical conditioning in fact does not exist. In other words when a conditioned stimulus is presented without unconditioned stimulus for many trials, conditioned response doesn't stop; rather the strength of it rather increases. Eysenck posits that this phenomenon is also the cause of anxiety. He calls this as "Incubation".

5. Humanistic model: This model posits that when there is significant disparity between ideal and real-self, the chances of anxiety increases. Incongruence leads to anxiety. The model 


\section{Psychological Models of Depression and Anxiety: Counselor's Perspectives}

posits that when human motive to self-actualize oneself is blocked because of external barriers, anxiety sets in. In other words, when a person's striving toward psychological growth is blocked, anxiety occurs.

6. Existential model: Existential model posits that basic human motive is "Will to meaning" and when this motive is not being fulfilled a person experiences existential frustration and it then lead to anxiety. It is also called as “Existential vacuum”.

7. Two-factor model: Two - factor theory was given by Mowrer in 1947. The basic idea of the model posits that both classical and instrumental learning are responsible for causing anxiety. The model goes on to explain that anxiety is learned through classical learning while it is maintained through instrumental learning.

8. Cognitive model: Cognitive theories posit that appraisal plays the pivotal role in anxiety. The appraisal process involves two stages, a primary appraisal in which an individual determines the threat posed by the environment and following a threatening primary appraisal a secondary appraisal where the individual evaluates his or her ability to cope with the demand imposed by the environment. Beck and Emery (1985) suggests that maladaptive anxiety results from distortions in the appraisal process. People who suffer from chronic anxiety are those who misperceive benign situations as threatening. Barlow (1988) extends the appraisal model and suggests that anxiety is cognitive-affective phenomena, at the core of which is negative affect. Perceptions of threat are influenced by early experiences with uncontrollability that creates a psychological vulnerability to anxiety and a biological disposition to experience anxiety in the face of negative events. Specifically, negative events; activate the biological vulnerability to stress, leading trait-anxious individuals to perceive the environment as threatening even in the absence of identifiable stressor.

9. Meta-cognitive model: Wells (2009) developed the metacognitive model of generalized anxiety disorder (GAD). This model focuses on the role of metacognitive beliefs (i.e., thoughts about thinking) in the development and maintenance of emotional disorders and suggests that positive beliefs about the benefits of worry (e.g., "Worrying helps me cope') and negative beliefs about the danger and uncontrollability of worry (e.g., "my worrying is bad for me”) are associated with pathological worry. This model has led to the development of new treatments for pathological worry, with research suggesting that modifying beliefs about worry enhances treatment outcome in anxiety disordered adults (Wells \& King 2006).

\section{Worry and Generalized anxiety Disorder:}

Worry has been described as "a chain of thoughts and images, negatively affect-laden and relatively uncontrollable”' (Borkovec et al. 1983, p. 10). Similarly, Vasey and Daleiden (1994, p. 186) describe worry as "primarily an anticipatory cognitive process involving repetitive, primarily verbal thoughts related to possible threatening outcomes and their potential consequences." Worry is also a feature of other anxiety disorders, including separation anxiety disorder and social phobia (Perrin \& Last 1997). For example, a child with separation anxiety disorder may worry about losing or separating from his or her parent, and so may have 


\section{Psychological Models of Depression and Anxiety: Counselor's Perspectives}

difficulties attending school or sleeping on his or her own . Clinical worry is also associated with risk of comorbidity with other anxiety disorders and depression.

\section{The role of metacognition in worry}

Wells (2004) defined metacognition as "the cognitive processes, strategies, and knowledge that are involved in the regulation and appraisal of thinking itself'” (p. 167). Vasey (1993, p. 23) suggests that metacognition “involves introspective knowledge about (1) one's cognitive states and abilities and their operation, and (2) strategies and procedures for effective problem solving", and is “the nonconscious operations of a central executive that organizes and guides cognitive activity such as problem solving'. The main focus of the model has been on the metacognitive factors associated with pathological worry in adults with GAD. Wells ( 2009) hypothesized that worry is maintained by metacognitive beliefs concerning the benefits and dangers of worrying. Initially, it is is triggered as a coping response by an intrusive thought (e.g., "What if I get cancer?') and is primarily focused on a range of issues including physical health, social, or financial concerns. This is known as Type 1 worrying. Positive metacognitive beliefs are linked to the usefulness of worry as a coping strategy, and these beliefs include "Worrying helps me cope", or "If I worry I'll be prepared”. Wells suggests that positive beliefs about worry are normal and should be observed in clinical and non-clinical populations.

Individuals with GAD are differentiated by the activation of negative beliefs about worry, specifically the uncontrollability (e.g., "My worrying thoughts persist, no matter how I try to stop them') and the danger (e.g., 'Worrying will make me go crazy') of worry. The activation of these beliefs contributes to negative appraisals of worry, including worrying about worry, which is known as Type 2 worry or meta-worry. Negative emotions associated with meta-worry, such as increased anxiety, make it increasingly difficult for the individual to recognize that it is safe to stop worrying.

Wells (2009) posits that type 2 worry contributes to two feedback cycles that maintain the worry process. First, behaviors such as reassurance seeking or avoidance of cues that trigger worry maintain negative beliefs about the danger and uncontrollability of worry. This is because the individual relies on external information to control their thoughts, they miss out on opportunities to learn that worrying is controllable and harmless, and they are prevented from learning more adaptive coping strategies. The second feedback cycle relates to thought control strategies, such as suppression of thoughts that trigger worry. Thought-control strategies are hypothesized to actually increase the number of thought intrusions and reinforce the belief that worry is uncontrollable. There is also a failure to interrupt the worry process.

10. Trait model: McCrae and Costa in 1990 gave five factor model of personality. The model posits that human personality is mainly comprised of 5 main/core traits. These are openness to experience, conscientiousness, extraversion agreeableness and neuroticism. Earlier, Eysenck (1967) using a mixed trait-type factor analytical approach gave PEN model of personality and linked that to anxiety. PEN stands for Psychoticism, Extraversion and Neuroticism. 


\section{Psychological Models of Depression and Anxiety: Counselor's Perspectives}

11. Expressive-Behavioral model: Expressive-behavioral theories view anxiety as an innate response that has evolved because of its adaptive value. Izard (1991) conceptualizes anxiety as a composite of a number of discrete emotions and the most important of which is fear. Other emotions that combine with fear to create anxiety include anger, guilt and shame and which of these emotions combine with fear to create anxiety depends on the situation in which anxiety is expressed. Discrete emotions theorists focus on the affective or feeling component of anxiety while deemphasize the cognitive elements of anxiety.

12. Cloninger's Model: One latest model of personality and its relation to psychopathology has been proposed by Cloninger in the 1987. According to this model, the main traits are reward dependence, novelty seeking, harm avoidance and persistence. The three traits which he calls as characters are self-directedness, cooperativeness and self-transcendence. This is mainly biological model because it explains these traits in terms of neurotransmitters. This is measured by Temperament and Character Inventory (TCI). People high on harm avoidance dimension of their personality are prone to maladaptive anxiety.

13. Biological model: Biological theorists suggest that different emotions result from activation of specific parts of brain. One of the influential theories was given by Jeffery Gray in 1976.The theory asserts that anxiety stems from the activation of the "Behavioral-Activation System” (BIS). The BIS inhibits behavior in response to threatening stimuli. People who are high in trait-anxiety have very reactive BIS and hence are prone to anxiety. Behavioral genetics researchers have found that a substantial portion of the variability on measures of neuroticism can be attributed to genetic factors.

\section{DEPRESSION: NATURE AND MODELS}

Mood disorders are the category of mental disorders in which significant and chronic disruption in mood is the predominant symptom, causing impaired cognitive, behavioral and physical functioning. Major depression also known as simply depression is a mood disorder characterized by extreme and persistent feelings of despondency, worthlessness and hopelessness, causing impaired emotional, cognitive, behavioral and physical functioning. It is a complex diagnostic construct, applied to individuals with a particular set of symptoms among which the essential ingredients are a sad mood, a loss of interest in usual pleasurable activities and easy fatigability. Depression has substantial short term and long term consequences for the individuals affected their families and society. The symptoms can cause immense distress to patients and are often associated with a variety of social and occupational impairments. The social and economic burden it poses for the community makes it one of the most serious health problems faced by the society. The studies (Epidemiological Catchment Area Study) indicate that major depression has a 1 month prevalence of 2.2\% and life time prevalence of 5.8\% in Americans (Regier, 1988). In India, the prevalence rate for major depression was found to be 31.2 per 1000 (Murali, 2001). 


\section{Psychological Models of Depression and Anxiety: Counselor's Perspectives}

The symptoms in of depression in children and adolescents are often externalized and often expressed in the form of irritability and anger outburst. The diagnosis of depression as per ICD X is made in adults if the following core symptoms persist for two weeks.

- $\quad$ Persistent sad mood

- $\quad$ Easy fatigability and

- $\quad$ Loss of interest in usually pleasurable activities.

\section{MODELS OF DEPRESSION}

Depression is explained on the bio psychosocial model asserting that biological, psychological and social factors play a combined role in the etiology of depressive disorder. Here in this paper, only contemporary psychological models are discussed in brief.

1. Cognitive model: This model was propounded by Aaron Beck in 1967. He posits that after a series of negative events in childhood like death of a loved one, emotional or physical abuse, individuals may develop a deep seated negative schema, an enduring negative cognitive belief system about some aspect of life. People think negatively about themselves, their immediate world and their future. This combination is often known as cognitive triad. Beck argues that depression may result from a tendency to interpret everyday events in a negative way. These negative cognitive set of beliefs is known as cognitive errors. Some of the examples of these cognitive errors overgeneralization, minimization, magnification, personalization etc. In this theory, a self-blame schema is developed in which people feel personally responsible for every bad thing that happens in their life. In Beck's view, these cognitive errors and schemas are automatic and are not consciously available to the people and they are not even aware of thinking negatively and illogically. Thus, minor negative event can lead to a major depressive episode. In cognitive behaviour therapy, the patients are helped to be aware of these cognitive errors and start thinking more realistically and logically.

2. $\quad$ Learned helplessness model: This model was propounded by Martin Seligman in 1967. after his classic experiment on dogs. The basic idea of this model is depression occurs when people feel helpless and believe that whatever they do, it won't change the apparently negative and aversive situation based on their experiences. Later his theory was reformulated by Abramson et al in 1978 which suggests that people who are vulnerable to depression tend to offer internal, global and stable causal explanations for negative events.

3. Reinforcement model: This model was propounded by Peter Lewinsohn in 1973. It is a behavioral theory of depression. A key tenet of the approach is the assumption that a low rate of positive reinforcement constitutes a critical antecedent condition for the occurrence of depressive behaviors. In effect, the behavioral theory requires the onset of depression be accompanied by a reduction in positive reinforcement, that intensity of depression co vary with rate of positive reinforcement, and that improvement be accompanied by an increase of positive reinforcement. It has become evident that a low rate of behavior constitutes an extremely important clinical aspect of depression. Consistent with the major premises of the theory, the principal goal of

(C) The International Journal of Indian Psychology, ISSN 2348-5396 (e)| ISSN: 2349-3429 (p) | 122 


\section{Psychological Models of Depression and Anxiety: Counselor's Perspectives}

treatment is to restore an adequate schedule of positive reinforcement for the patient by altering the level, the quality, and the range of his activities and interpersonal interactions. According to Lewinsohn, depressed people are precisely those people who do not know how to cope with the fact that they are no longer receiving positive reinforcements like they were before. For example, a child who has newly moved to a new home and has consequently lost touch with prior friends might not have the social skills necessary to easily make new friends and could become depressed. Similarly, a man who has been fired from his job and encounters difficulty finding a new job might become depressed. The therapeutic implication of this model is to introduce activity scheduling techniques in psychotherapy.

4. Life event model:This model posits that significant negative life events and chronically stressful circumstances are associated with depression (Brown \& Harris, 1989). Negative life events and how people appraise and understand those events will decide whether the depression will occur or not.

5. Interpersonal models of depression: Negative interpersonal circumstances are particularly likely to play a role in depression. Marital, family and peer relations are often troubled and interpersonal forms of stress such as relationship endings, conflicts and lack of supportive relationships are consistently associated with depression (Beach \& Finchman, 1998). Subsequent to marital split, men faced a more heightened risk for developing depressive disorder than women. Interpersonal models of depression highlight how the disorder can be both a cause and a consequence of interpersonal problems. Coyne (1976) suggested that depressed people engage in behaviors that elicit rejection from others and this rejection leads to further depression. Depressed people generate interpersonal stress in their lives, which then makes them more depressed.

6. Social support model: In a landmark study, Brown and Harris (1978) first suggested the important role of social support in the onset of depression. In a study of large number of women who had experienced a serious life stress, they discovered that only $10 \%$ of the women, who had a friend in whom they could confide become depressed, compared to $37 \%$ of the women who did not have a close supporting relationship. Later prospective studies have confirmed the importance of social support or lack of it in predicting the later onset of depressive symptoms (Kendler et al, 2005).

7. Self-control model: Self-control model of depression was given by Lynn Rehm in 1977. It is based on the three processes included in a feedback loop model of self-control: selfmonitoring, self-evaluation, and self-reinforcement. In this model, depression is characterized as the result of deficits in these processes of self-control. Self-monitoring is described as the observation and evaluation of one's own behavior, including its antecedents (events preceding the behavior) and consequences. Rehm describes two characteristics of self-monitoring in those who experience depression: the tendency to attend only to negative events and the tendency to recognize only immediate consequences of behavior. Self-evaluation is described as an individual's perception of their growth and progress compared to an internal standard. For example, a dieter may compare their calorie count for the day to a goal and decide whether or not 


\section{Psychological Models of Depression and Anxiety: Counselor's Perspectives}

they reached that goal. An internal standard can be set by adopting externally imposed standards, such as a diet calorie chart based on sex and height, or they may choose criteria that is more or less stringent than external standards. In Rehm's model, self-evaluation is characterized in those experiencing depression by inaccurate, and often external, attributions of causality and stringent self-evaluation criteria. For example, an individual who self-imposes a criterion of obtaining $100 \%$ on every test they take has set an unrealistic criterion. When that stringent criteria is not met, the individual may inaccurately attribute their failure to an internal characteristic, such as intelligence. Rehm also includes self-reinforcement in his model, and states that those experiencing depression infrequently engage in self-reward and engage more frequently in selfpunishment.

8. Psychoanalytical model of depression: Psychoanalytic model of depression: This was given by Sigmund Freud in 1917. His theory posits that depression is a result of anger turns inward a process known as introjections which results due to the loss or rejection by a parent. Depression is a manifestation of inwardly directed anger and severe super ego demands. Freud distinguished between actual losses (e.g. death of a loved one) and symbolic losses (e.g. loss of a job). Both kinds of losses can produce depression by causing the individual to reexperience childhood episodes when they experienced loss of affection from some significant person (e.g. a parent). Later, Freud modified his theory stating that the tendency to internalize loss objects is normal, and that depression is simply due to an excessively severe super-ego. Thus, the depressive phase occurs when the individual's super-ego or conscience is dominant. In contrast, the manic phase occurs when the individual's ego or rational mind asserts itself, and $\mathrm{s}$ /he feels control. In order to avoid loss turning into depression, the individual needs to engage in a period of mourning work, during which s/he recalls memories of the lost one. This allows the individual to separate him/herself from the lost person, and so reduce the inner-directed anger. However, individuals very dependent on others for their sense of self-esteem may be unable to do this, and so remain extremely depressed.

9. Integrative model: This model says that depression is a function of multiple factors including biological, interpersonal, cognitive, behavioural and host of sociocultural influences. No single theory can account for the occurrence of depressive disorder in an individual.

\section{ANXIETY AND DEPRESSION: CO-MORBIDITY}

Research and clinical work has shown that clinically significant anxiety and depression often coexist. This is often as co-morbidity. It refers to the presence of more than one or two or more distinct clinical diagnosis in the same individual. One of the most common models which attempt to explain the existence of both depression and anxiety in clients is known as tripartite model given by Clark and Watson in 1991. The tripartite model states that anxiety and depression share a common Negative Affectivity (NA) factor, whereas physiological hyperarousal (PH) is specific to anxiety and low positive affect (PA) is specific to depression. NA may involve "transdiagnostic factors," including neuroticism, rumination, and intolerance of 


\section{Psychological Models of Depression and Anxiety: Counselor's Perspectives}

uncertainty. The shared NA factor links sadness/depression and anxiety/fearfulness, thus accounting for co morbidity between anxiety and depression.

Latest class analysis, a statistical procedure similar to factor analysis examined the commonality among anxiety and depression. A latent class analysis indicated that the vast majority of adolescents with high levels of depression also had high levels of anxiety, but that the reverse was not true. Adolescents with high levels of anxiety had either high or low levels of depression (Ferdinand, De Nijs, van Lier, \& Verhulst, 2005). This indicates that depressed youth tend to report high levels of both depression and anxiety, whereas anxious youth tend to report high levels of anxiety but relatively low levels of depression. One of the models explaining frequent co-occurrence of anxiety and depression was given by Seligman and Ollendick (1998). They argued possibility of four factors: (1) co morbidity between anxiety and depression in youth is due to overlap in definitions; (2) anxiety and depression represent two indicators of a single construct; (3) comorbidity is due to overlap in risk factors; (4) anxiety causes or puts youth at risk for depression. There are two concepts which are important to remember in understanding developmental psychopathology among adolescents.

These are

a) Principles of equifinality: It means that different pathways or risk factors may lead to the same outcome. It means that the same clinical condition/psychopathology might be caused by different factors. For example, depression might occur because of lack of social skill deficit or it may also occur because of marital discord and

b) Principle of multifinality: It means the same risk factor may lead to different outcomes in examining the interactive processes that may lead to the emergence of psychopathology. For example, marital discord may lead to depression or it may also lead to post traumatic stress disorder.

\section{Simple scientific ways of managing clinically significant anxiety}

There are many psychological strategies which might help to deal with excessive anxiety. It is important to remember that diagnosis be done by trained mental professional and patients learn the skills of anxiety management by these trained professionals.

The following techniques might be useful

1. Understanding the specific possible causes of anxiety is the key to learn the management skills

2. Deep breathing technique

3. Jacobson Progressive Muscular Relaxation

4. Mindfulness

5. Learning to learn facing the threatening situations after acquiring the required skills 


\section{Psychological Models of Depression and Anxiety: Counselor's Perspectives}

\section{Simple scientific ways of managing sad mood}

1. Making a good day diary in which any event which improves one's mood need to be written. The possible cause of why the event was pleasurable/good should be either written or discussed

2. Trying to engage in activities which are being liked. For example listening to songs, walking etc. The activity may be done for short period of time without.

3. Involving in religious and spiritual practices as much as possible.

4. Practicing mindfulness

5. Walking or jogging

\section{CONCLUSIONS}

Negative psychological states like anxiety and depression are commonly experienced by children, youths and adults. Negative life events often cause people to experience threat and sad mood which are often adaptive and people come out with them with time. However, there are times the intensity, frequency and duration of threat and sad mood persist longer than the usual duration of adaptation and start effecting people's social, occupational, interpersonal and biological functioning. These need to evaluated and managed by trained mental health professionals. Co-morbid anxiety and depression are very common among patients of these clinical conditions. Clinicians need to make sure that co-morbidity be properly examined and treatment is modeled based on this. It will help proper management of the patients and increase the quality of clinical outcome and there by patients' well-being.

\section{Acknowledgments}

The author appreciates all those who participated in the study and helped to facilitate the research process.

\section{Conflict of Interests}

The author declared no conflict of interests.

\section{REFERENCES}

Barlow, D. H. (1988). Anxiety and its disorders: The nature and treatment of anxiety and panic. New York: Guilford.

Beach, S.R.H. \& Fincham, F.D.(1998). Marital process and depression in L.L. Abate (ed.), Family psychopathology: The relational roots of dysfunctional behaviour, Guilford press, New York.

Beck, A. T., \& Emery, G. (1985). Anxiety disorders and phobias: A cognitive perspective. New York: Basic Books.

Beck, A.T. (1967). The diagnosis and management of depression. Philadelphia, PA: University of Pennsylvania Press. 


\section{Psychological Models of Depression and Anxiety: Counselor's Perspectives}

Borkovec, T., Robinson, E., Pruzinsky, T., \& DePree, J. A. (1983). Preliminary exploration of worry: Some characteristics and processes. Behaviour Research and Therapy, 21, 9-16.

Bowlby, J. (1969). Attachment. Attachment and loss: Vol. 1. Loss. New York: Basic Books.

Brown, G.W. \& Harris, T.O. (1978). Social Origins of Depression: A Study of Psychiatric Disorder in Women. New York (NY): The Free Press.

Clark, L.A., \& Watson, D. (1991). Tripartite model of anxiety and depression: Psychometric evidence and taxonomic implications. Journal of Abnormal Psychology, 100, 316-336.

Cloninger, C. R. (1987). A systematic method for clinical description and classification of personality variants. Archives of General Psychiatry, 44, 573-588.

Coyne, J. C. (1976).Depression and the response of others. Journal of Abnormal Psychology, 85(2), 186-193.

Eysenck, H. J. (1968). A theory of the incubation of anxiety/fear responses. Behavior Research and Therapy, 6, 309-321.

Ferdinand, R.F, de Nijs, P.F.A, van Lier P., \& Verhulst, F.C. (2005). Latent class analysis of anxiety and depressive symptoms in referred adolescents. Journal of Affective Disorders, 88, 299-306.

Freud, S. (1926). Inhibitions, symptoms and anxiety. 1975 In the standard edition in the complete psychological work of Sigmund Freud. London: Hogarth Press.

Freud, S. (1917). Mourning and melancholia. Standard edition

Gray, J.A. (1976). The behavioural inhibition system: A possible substrate for anxiety. In M.P. Feldman and A.M. Broadhurst (eds), Theoretical and Experimental Bases of Behaviour Modification. London: Wiley. pp. 3-41.

Izard, C. E. (1991). The psychology of emotions. New York: Plenum.

Kendler, K.S, Kuhn, J.W, Vittum, J, Prescott, C.A, Riley, B.(2005). The interaction of stressful life events and a serotonin transporter polymorphism in the prediction of episodes of major depression: a replication. Archives of General Psychiatry.62(5), 529-535.

Lewinsohn, P. \& Graf, M. (1973). Pleasant activities and depression. Journal of Consulting and Clinical Psychology,41(2), 261-268.

May, R. (1950). The meaning of anxiety. New York.

McCrae. R. R., \& Costa, P. T.. Jr. (1990). Personality' in adulthood. New York: Guilford.

MOWRER, O. H. (1947). On the dual nature of learning-a reinterpretation of conditioning and problem solving. Harvard Educational Review, 17, 102-148.

Murali, M. S. (2001). Epidemiological Study of Prevalence of Mental Disorders in India. Indian Journal of Community Medicine, 26, 198-200.

Perrin, S., \& Last, C. G. (1997). Worrisome thoughts in children clinically referred for anxiety disorder. Journal of Clinical Child Psychology, 26, 181-189.

Regier, D.A., Boyd, J.H., Burke, J.D. Jr., Rae, D. S., Myers, J.K., Kramer, M., Robins, L.N., George, L.K., Karno M., Locke B.Z. (1988). One month prevalence of mental-disorders in the United States: based on five epidemiologic Catchment area sites. Archives of General psychiatry,45, 977-986. 


\section{Psychological Models of Depression and Anxiety: Counselor's Perspectives}

Rehm, L. P. (1977). A self-control model of depression. Behavior Therapy, 8(5), 787-804 Seligman, L.D, Ollendick., \& T. H.(1998). Comorbidity of anxiety and depression in children and adolescents: An integrative review. Clinical Child and Family Psychology Review,1, 125-144.

Seligman, M.E.P. (1967). "Effects of inescapable shock upon subsequent escape and avoidance responding". Journal of Comparative and Physiological Psychology 63 (1), 28-33.

Strelau, J., \& Zawadzki, B. (1997). Temperament and personality: Eysenck's three super factors as related to temperamental dimensions. In H. Nyborg (Ed.), The scientific study of human nature: Tribute to Hans J. Eysenck at eighty (pp. 68-91). New York: Pergamon.

Vasey, M. W. (1993). Development and cognition in childhood anxiety: The example of worry. Advances in Clinical Child Psychology, 15, 1-39.

Vasey, M. W., \& Daleiden, E. L. (1994). Worry in children. In G. C. L. Davey \& F. Tallis (Eds.), Worrying: Perspectives on theory, assessment and treatment (pp. 185-207). Oxford, England: Wiley.

Wells, A. (2004). A cognitive model of GAD: Metacognitions and pathological worry. In R. G. Heimberg, C. L. Turk, \& D. S. Mennin (Eds.), Generalized anxiety disorder: Advances in research and practice (pp. 164-186). New York: The Guildford Press.

Wells, A. (2009). Metacognitive therapy for anxiety and depression. New York, NY: The Guildford Press.

Wells, A., \& King, P. (2006). Metacognitive therapy for generalized anxiety disorder: An open trial. Journal of Behavior Therapy and Experimental Psychiatry, 37, 206-212.

Zuckerman, M., \& Como, P. (1983). Sensation-seeking and arousal systems. Personality and Individual Differences, 4, 381-386.

How to cite this article: Chouhan V, Sharma P (2017), Psychological Models of Depression and Anxiety: Counselor's Perspectives, International Journal of Indian Psychology, Volume 4, Issue 2, No. 85, ISSN:2348-5396 (e), ISSN:2349-3429 (p), DIP:18.01.015/20170402, ISBN: 978-1365-68608-5 